Supplement of Solid Earth, 10, 1707-1715, 2019 https://doi.org/10.5194/se-10-1707-2019-supplement (C) Author(s) 2019. This work is distributed under the Creative Commons Attribution 4.0 License.

(c) (1)

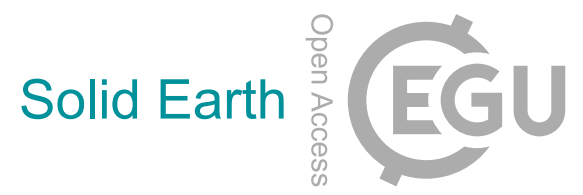

Supplement of

\title{
Uncertainty in regional estimates of capacity for carbon capture and stor- age
}

Mark Wilkinson and Debbie Polson

Correspondence to: Mark Wilkinson (mark.wilkinson@ed.ac.uk)

The copyright of individual parts of the supplement might differ from the CC BY 4.0 License. 
Supplementary Information, Wilkinson and Polson

Table 1 - Data used for calculation of storage capacity

\begin{tabular}{|c|c|c|c|c|c|c|}
\hline Storage unit & $\begin{array}{l}\text { Surface } \\
\text { area } / \mathrm{m}^{2}\end{array}$ & $\begin{array}{l}\text { Thickness } \\
\text { / m }\end{array}$ & $\begin{array}{l}\text { Volume * } \\
/ \mathrm{m}^{3}\end{array}$ & N:G & Porosity & $\begin{array}{l}\text { Storage volume } \\
/ \mathrm{Mt} \mathrm{CO}_{2}\end{array}$ \\
\hline Burns Sandstone Member & & & $1.51 \mathrm{E}+12$ & 0.75 & 0.18 & 2650 \\
\hline Burns Sandstone Member & & & $9.15 \mathrm{E}+11$ & 0.5 & 0.23 & 1368 \\
\hline Burns Sandstone Member & $2.25 \mathrm{E}+09$ & 435 & & 0.8 & 0.24 & 2443 \\
\hline Burns Sandstone Member & $2.03 \mathrm{E}+09$ & 885 & & 0.85 & 0.15 & 2969 \\
\hline Burns Sandstone Member & $1.80 \mathrm{E}+09$ & 400 & & 0.7 & 0.15 & 983 \\
\hline Burns Sandstone Member & $2.03 E+09$ & 43 & & 0.7 & 0.15 & 119 \\
\hline Burns Sandstone Member & $2.70 \mathrm{E}+09$ & 876 & & 0.7 & 0.25 & 5381 \\
\hline Burns Sandstone Member & $1.37 \mathrm{E}+09$ & 150 & & 1 & 0.23 & 612 \\
\hline Beatrice Formation & $2.70 \mathrm{E}+09$ & 30 & & 0.5 & 0.16 & 84 \\
\hline Beatrice Formation & $1.80 \mathrm{E}+09$ & 54 & & 0.8 & 0.15 & 152 \\
\hline Beatrice Formation & & & $1.30 \mathrm{E}+11$ & 0.71 & 0.16 & 192 \\
\hline Beatrice Formation & $1.80 \mathrm{E}+09$ & 48 & & 0.87 & 0.16 & 156 \\
\hline Beatrice Formation & $1.60 \mathrm{E}+09$ & 30 & & 0.7 & 0.16 & 70 \\
\hline Beatrice Formation & $1.81 \mathrm{E}+09$ & 45 & & 0.71 & 0.16 & 120 \\
\hline Beatrice Formation & $2.70 \mathrm{E}+09$ & 48 & & 0.71 & 0.16 & 191 \\
\hline Beatrice Formation & $2.03 E+09$ & 48 & & 0.77 & 0.16 & 156 \\
\hline Beatrice Formation & $1.35 \mathrm{E}+09$ & 48 & & 0.63 & 0.16 & 85 \\
\hline Beatrice Formation & & & $9.93 \mathrm{E}+10$ & 0.65 & 0.13 & 109 \\
\hline Beatrice Formation & $1.80 \mathrm{E}+09$ & 10 & & 1 & 0.16 & 39 \\
\hline Orrin Formation & $1.35 \mathrm{E}+09$ & 43 & & 0.7 & 0.14 & 74 \\
\hline Orrin Formation & $1.24 \mathrm{E}+09$ & 58 & & 0.7 & 0.13 & 85 \\
\hline Orrin Formation & $1.24 \mathrm{E}+09$ & 53 & & 0.96 & 0.175 & 143 \\
\hline Orrin Formation & & & $5.75 \mathrm{E}+11$ & 0.75 & 0.14 & 785 \\
\hline Orrin Formation & $1.13 \mathrm{E}+09$ & 58 & & 0.75 & 0.15 & 95 \\
\hline Orrin Formation & $1.13 \mathrm{E}+09$ & 40 & & 0.75 & 0.15 & 66 \\
\hline Orrin Formation & $1.28 \mathrm{E}+09$ & 60 & & 0.75 & 0.15 & 112 \\
\hline Orrin Formation & $1.13 \mathrm{E}+09$ & 51 & & 0.75 & 0.14 & 79 \\
\hline Orrin Formation & $1.35 \mathrm{E}+09$ & 54 & & 0.78 & 0.15 & 111 \\
\hline Orrin Formation & $1.24 \mathrm{E}+09$ & 53 & & 0.96 & 0.175 & 143 \\
\hline Orrin Formation & $2.09 \mathrm{E}+09$ & 54 & & 0.75 & 0.14 & 154 \\
\hline Orrin Formation & & & $6.90 \mathrm{E}+10$ & 0.75 & 0.14 & 94 \\
\hline Orrin Formation & $1.13 \mathrm{E}+09$ & 8 & & 1 & 0.15 & 17 \\
\hline Mains Formation & $4.95 E+09$ & 40 & & 0.4 & 0.15 & 154 \\
\hline Mains Formation & & & $1.89 \mathrm{E}+11$ & 0.58 & 0.15 & 214 \\
\hline Mains Formation & $3.83 \mathrm{E}+09$ & 40 & & 0.6 & 0.15 & 179 \\
\hline Mains Formation & $4.05 E+09$ & 30 & & 0.4 & 0.15 & 95 \\
\hline Mains Formation & $3.98 \mathrm{E}+09$ & 40 & & 0.65 & 0.15 & 202 \\
\hline Mains Formation & $4.73 E+09$ & 40 & & 0.58 & 0.15 & 214 \\
\hline Mains Formation & $4.05 E+09$ & 40 & & 0.61 & 0.15 & 193 \\
\hline Mains Formation & $3.96 \mathrm{E}+09$ & 40 & & 0.45 & 0.15 & 139 \\
\hline Mains Formation & & & $1.60 \mathrm{E}+11$ & 0.68 & 0.15 & 212 \\
\hline Mains Formation & $4.05 E+09$ & 31 & & 1 & 0.15 & 248 \\
\hline Hopeman Sandstone Fm & $3.04 \mathrm{E}+09$ & 75 & & 0.7 & 0.22 & 456 \\
\hline
\end{tabular}




\begin{tabular}{|c|c|c|c|c|c|c|}
\hline Hopeman Sandstone Fm & $1.80 \mathrm{E}+09$ & 79 & & 1 & 0.16 & 296 \\
\hline Hopeman Sandstone Fm & & & $2.79 \mathrm{E}+11$ & 0.95 & 0.1 & 345 \\
\hline Hopeman Sandstone $\mathrm{Fm}$ & $1.80 \mathrm{E}+09$ & 80 & & 0.99 & 0.07 & 130 \\
\hline Hopeman Sandstone Fm & $1.58 \mathrm{E}+09$ & 40 & & 1 & 0.15 & 123 \\
\hline Hopeman Sandstone Fm & $1.80 \mathrm{E}+09$ & 80 & & 1 & 0.15 & 281 \\
\hline Hopeman Sandstone Fm & $3.04 \mathrm{E}+09$ & 78 & & 0.99 & 0.12 & 366 \\
\hline Hopeman Sandstone Fm & $1.80 \mathrm{E}+09$ & 79 & & 0.99 & 0.07 & 128 \\
\hline Hopeman Sandstone Fm & $1.80 \mathrm{E}+09$ & 79 & & 1 & 0.16 & 296 \\
\hline Hopeman Sandstone Fm & $1.62 \mathrm{E}+09$ & 79 & & 0.98 & 0.15 & 245 \\
\hline Hopeman Sandstone Fm & $1.58 \mathrm{E}+09$ & 79 & & 0.99 & 0.12 & 192 \\
\hline Hopeman Sandstone Fm & $1.70 \mathrm{E}+09$ & 22.86 & & 1 & 0.225 & 114 \\
\hline Findhorn Formation & 4.39E+09 & 612 & & 0.8 & 0.13 & 3630 \\
\hline Findhorn Formation & $1.80 \mathrm{E}+09$ & 590 & & 0.9 & 0.23 & 2858 \\
\hline Findhorn Formation & $1.80 \mathrm{E}+09$ & 600 & & 0.99 & 0.075 & 1042 \\
\hline Findhorn Formation & & & $1.55 \mathrm{E}+12$ & 0.74 & 0.15 & 2237 \\
\hline Findhorn Formation & $1.58 \mathrm{E}+09$ & 366.5 & & 0.5 & 0.15 & 563 \\
\hline Findhorn Formation & $1.68 \mathrm{E}+09$ & 400 & & 0.98 & 0.1633 & 1398 \\
\hline Findhorn Formation & $1.78 \mathrm{E}+09$ & 452 & & 0.98 & 0.075 & 769 \\
\hline Findhorn Formation & $2.36 \mathrm{E}+09$ & 452 & & 0.94 & 0.15 & 1955 \\
\hline Findhorn Formation & $1.58 \mathrm{E}+09$ & 452 & & 0.98 & 0.15 & 1360 \\
\hline Findhorn Formation & $1.80 \mathrm{E}+09$ & 600 & & 0.99 & 0.075 & 1042 \\
\hline Findhorn Formation & $1.87 \mathrm{E}+09$ & 415 & & 0.5 & 0.23 & 1159 \\
\hline Findhorn Formation & $1.80 \mathrm{E}+09$ & 487.68 & & 1 & 0.225 & 2568 \\
\hline Strath Rory Formation & $2.70 \mathrm{E}+09$ & 369.5 & & 0.8 & 0.08 & 830 \\
\hline Strath Rory Formation & $1.62 \mathrm{E}+09$ & 400 & & 0.5 & 0.15 & 632 \\
\hline Strath Rory Formation & $1.80 \mathrm{E}+09$ & 600 & & 0.99 & 0.075 & 1042 \\
\hline Strath Rory Formation & & & $1.63 \mathrm{E}+11$ & 0.9 & 0.08 & 153 \\
\hline Strath Rory Formation & $1.69 \mathrm{E}+09$ & 576 & & 0.25 & 0.17 & 537 \\
\hline Strath Rory Formation & $1.35 \mathrm{E}+09$ & 350 & & 0.9 & 0.07 & 387 \\
\hline Strath Rory Formation & $1.54 \mathrm{E}+09$ & 500 & & 0.48 & 0.1 & 481 \\
\hline Strath Rory Formation & $2.36 \mathrm{E}+09$ & 687 & & 0.9 & 0.08 & 1518 \\
\hline Strath Rory Formation & $2.03 E+09$ & 687 & & 0.39 & 0.17 & 1199 \\
\hline Strath Rory Formation & $1.58 \mathrm{E}+09$ & 250 & & 0.99 & 0.15 & 760 \\
\hline Strath Rory Formation & $1.58 \mathrm{E}+09$ & 686 & & 0.88 & 0.08 & 989 \\
\hline Strath Rory Formation & $1.58 \mathrm{E}+09$ & 686 & & 0.96 & 0.17 & 2292 \\
\hline Strath Rory Formation & $9.00 \mathrm{E}+08$ & 43 & & 1 & 0.15 & 75 \\
\hline
\end{tabular}

* Volume is only given where area and thickness were not listed separately. 